\title{
Recurrent Fallopian Tube Serous Adenocarcinoma
}

National Cancer Institute

\section{Source}

National Cancer Institute. Recurrent Fallopian Tube Serous Adenocarcinoma. NCI

Thesaurus. Code C153613.

The reemergence of fallopian tube serous adenocarcinoma after a period of remission. 